\title{
Epigenetic epidemiology: is there cause for optimism?
}

\author{
"Statistical and modeling approaches to data analyses need \\ to develop further to align with the complexity and ever changing \\ nature of living organisms"
}

Keywords: complex traits $\bullet$ DNA methylation $\bullet$ epigenetic epidemiology $\bullet$ epigenetics - EWAS • noncommunicable diseases

Modulation of gene expression is influenced by environmental exposures, genetic variation and stochastic processes. Epigenetic biomarkers that signal the outcome of their combined impact could be important early predictors of susceptibility to chronic noncommunicable diseases. Can epigenetic epidemiology shed light on causality and mechanism?

Common complex traits arise over time and are the end result of lifestyle choices and a combination of environmental exposures during periods of developmental vulnerability and epigenetic plasticity in the presence of a combination of susceptible genetic variants. Genome-wide association studies to identify the genetic contributions to complex traits have identified many high probability allelic associations, but almost invariably each with a small effect, explaining only a modest percentage of the heritability of the trait. Notably, many estimates of heritability are inflated and do not adequately take into account-shared environment and other familial factors.

It is not new to suggest that tissue-specific epigenetic modulation plays a major role in the development of complex traits, yet few have managed to support this using a robust experimental approach. Whereas the genome remains constant, gene expression is labile, demonstrates cell type and developmental specificity, age and sex differences, and is variably responsive to changes in the environment.

What are the relationships between genetic variation and different environments that influence epigenetic responses? How do we begin to understand causal relationships that influence gene expression? From an experimental perspective, it is challenging to design studies that can address these questions, yet present a feasible and affordable approach. Large international and interdisciplinary studies are beginning to provide a good framework to better understand gene expression regulation in humans. Though open to criticism, their value should not be underestimated. They include ENCODE that examined 147 cell lines to search for functional elements in the genome [1]; the Epigenomics Roadmap [2] that examined 127 tissue biopsies from embryos and adults, both diseased and healthy, with a focus on identifying functional regulatory elements; the International Human Epigenome Consortium [3]; and the Genome Tissue Expression project (GTEx) that explores the relationship between genetic variation and tissue-specific gene expression and alternate splicing by sequencing whole genomes and examining gene expression in over 20,000 tissue samples from 900 individuals [4]. These data are beginning to give insight into cell specific, developmental stage, sex, age and ethnicity influenced, and stochastic gene expression differences at an individual and population level. The layered annotations in genome browsers highlight the importance of integrating transcriptome data (mRNA, miRNA and lncRNA) with DNA methylation, histone modification and chromatin interaction data to more fully understand function.

Epigenome-wide association studies (EWASs) to identify regulatory mechanisms

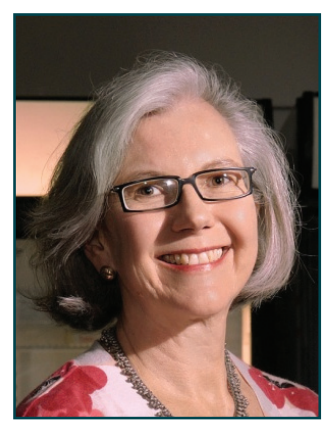

Michèle Ramsay

Sydney Brenner Institute for Molecular Bioscience, University of the Witwatersrand, Room 109, The Mount, 9 Jubilee Road, Parktown, 2050, Gauteng, Johannesburg, South Africa michele.ramsay@wits.ac.za 
and to predict their causal link to common diseases [5] currently make use of a crude proxy by examining percentage methylation differences at $-2 \%$ of $\mathrm{CpG}$ sites in the genome, sites selected in part for their accessibility using available detection methods (viz. HumanMethylation450k BeadChip Array [6]). Increasingly, it is accepted that individual $\mathrm{CpG}$ sites may not provide as much information as multiple $\mathrm{CpG}$ sites (differentially methylated regions). Several excellent reviews highlight the limitations and challenges of using archival samples from longitudinal cohort studies for epigenetic epidemiology [7-10]. These DNA samples usually originate from blood, saliva or buccal cells and essentially many challenges remain as we grapple to understand which tissues and cell types would be most appropriate to investigate, what types of epigenetic changes we should be looking for, what effect sizes would be likely to have physiological impact (and therefore guide the statistical assessment of sample size for the studies), and whether we are looking at all the epigenetic mechanisms that matter.

Cellular heterogeneity in peripheral blood is recognized as a confounder in epigenetic disease and trait association studies [11,12]. Although bioinformatics algorithms have been developed to infer the cellular mix, this is not ideal [13]. Two examples of successful epigenetic studies are highlighted, one for an environmental exposure and the other for a common trait.

Locus-specific associations of environmentally linked epigenetic changes have been described for in utero exposure to tobacco and adult smoking [14,15]. The AHRR locus methylation profile has been described as an epigenetic marker for tobacco exposure. Interestingly, it shows a degree of ethnic specificity when compared between European and southeast Asian populations, is sensitive to the cellular composition of the blood and is likely influenced by sex and age [16]. It provides an opportunity to study objectively tested tobacco exposure with smoking-related outcomes, such as cancer and cardiovascular disease.

A recent systematic review assessed our current knowledge about the link between epigenetics and obesity [17] and pointed out that some epigenetic markers for childhood obesity, in this case DNA methylation at the RXRA locus, could be detected at birth, providing opportunities for early intervention [18]. What is important from an intervention perspective is that epigenetic markers such as DNA methylation and histone modifications are malleable and some of them can be altered in adult life through lifestyle changes, to result in a more favorable antiobesogenic profile in specific tissues [19].

Epigenetic studies in humans remain limited for obvious practical and ethical considerations. Is there cause for optimism in unraveling complex epigenetic mechanisms for late onset chronic conditions with noncommunicable elements? Could this lead to improved prevention, prediction and treatment? Like epidemiological studies, epigenetic studies will be fraught with bias, confounding, reverse causation and bidirectional influences. However, using longitudinal cohorts and epidemiological approaches to epigenetics, is likely to yield robust findings that can be replicated [20]. Statistical and modeling approaches to data analyses need to develop further to align with the complexity and ever changing nature of living organisms. The signal-tonoise ratio in genome-wide approaches to data generation and discovery remains a significant impediment to understanding biological processes, with abundant false-positive and false-negative signals. Good advice urges an approach for an integrated functional study design to begin to understand molecular mechanisms in diseases, including epigenetic modulation [7]. Statistical deconvolution of tissues with mixed cell types would make legacy DNA sample collections more valuable, but their tissues of origin may still not be appropriate for the phenotype under investigation. The relatively recent and welcome focus on biobanking of specimens for future research will not only greatly enhance the ability to do epigenetic epidemiology, but underscores the need for sustainable infrastructure and funding to plan for future research. What is the subject of much debate is whose responsibility this should be, the individual researchers, the academic institutions, research funders, government bodies or other stakeholders.

It is already feasible to identify environmental causes of specific cancers by studying the mutation profiles in somatic tumor tissue [21]. For example, CC to TT transitions at pyrimidine dimers are signatures of ultraviolet light exposure in nonmelanoma skin cancer, aflatoxin exposure is associated with a TP53 codon 249 mutation in hepatocellular carcinoma and $\mathrm{G}>\mathrm{T}$ transversions at methylated CpG sites are characteristic of exposure to tobacco. We are beginning to detect epigenetic signals for exposure (e.g., smoking) and as markers for phenotype (e.g., obesity). When robust epigenetic and geneexpression biomarkers for risk are developed for diseases of lifestyle, more effective intervention strategies could be developed.

\section{Conclusion}

As new studies are planned and others are enriched with waves of data and biological specimen collection, it would be prudent to collect and prepare samples for future epigenetic studies. However, it is not a simple task to choose the appropriate approach and it will be an expensive addition to grant applications. Although we still lack adequate knowledge to fully inform our decisions, it is clear that cell type-specific studies are 
important, as tissues include many different cell types in different ratios and resulting data are therefore difficult to interpret. Fortunately, technologies are developing to assist data generation from small samples, even to the level of individual cells. Now that we have created an expectation that epigenetic signals are amenable to therapeutic manipulation, it is important to manage the expectations of the health professionals and of the patients during this important discovery phase.

\section{Future perspective}

Understanding the mechanisms that regulate gene expression and function provides an opportunity for targeted therapeutic intervention. However, its impact on reducing the health burden of common noncommunicable diseases will only be apparent when we develop a deeper understanding of the complex interrelationships between environment, behavior, physi- ological response and genetic predisposition. With increasing knowledge comes optimism.

\section{Disclaimer}

The views expressed do not necessarily represent the official views of the National Research Foundation of South Africa. The content is solely the view of the author and does not necessarily represent the official views of the $\mathrm{NIH}$.

\section{Financial \& competing interests disclosure}

M Ramsay is supported by the South African Research Chairs Initiative of the Department of Science and Technology and National Research Foundation (NRF) of South Africa. The author has no other relevant affiliations or financial involvement with any organization or entity with a financial interest in or financial conflict with the subject matter or materials discussed in the manuscript apart from those disclosed.

No writing assistance was utilized in the production of this manuscript.

\section{References}

1 Consortium EP. An integrated encyclopedia of DNA elements in the human genome. Nature 489(7414), 57-74 (2012).

2 Epigenomics roadmap. www.nature.com/epigenomeroadmap

3 Time for the epigenome. Nature 493(7281), 587 (2010).

4 Genome tissue expression project. www.gtexportal.org

5 Rakyan VK, Down TA, Balding DJ, Beck S. Epigenome-wide association studies for common human diseases. Nat. Rev. Genet. 12(8), 529-541 (2011).

6 Sandoval J, Heyn H, Moran S et al. Validation of a DNA methylation microarray for $450,000 \mathrm{CPG}$ sites in the human genome. Epigenetics 6(6), 692-702 (2014).

7 Mill J, Heijmans BT. From promises to practical strategies in epigenetic epidemiology. Nat. Rev. Genet. 14(8), 585-594 (2013).

8 Heyn H, Esteller M. DNA methylation profiling in the clinic: applications and challenges. Nat. Rev. Genet. 13(10), 679-692 (2012).

$9 \quad \mathrm{Ng}$ JW, Barrett LM, Wong A, Kuh D, Smith GD, Relton CL. The role of longitudinal cohort studies in epigenetic epidemiology: challenges and opportunities. Genome Biol. 13(6), 246 (2012).

10 Bakulski KM, Fallin MD. Epigenetic epidemiology: promises for public health research. Environ. Mol. Mutat. 55(3), 171-183 (2014).

11 Wu HC, Delgado-Cruzata L, Flom JD et al. Global methylation profiles in DNA from different blood cell types. Epigenetics 6(1), 76-85 (2011).
12 Reinius LE, Acevedo N, Joerink M et al. Differential DNA methylation in purified human blood cells: implications for cell lineage and studies on disease susceptibility. PLOS ONE 7(7), e41361 (2012).

13 Houseman EA, Accomando WP, Koestler DC et al. DNA methylation arrays as surrogate measures of cell mixture distribution. BMC Bioinformatics 13, 86 (2012).

14 Joubert BR, Haberg SE, Nilsen RM et al. 450k epigenomewide scan identifies differential DNA methylation in newborns related to maternal smoking during pregnancy. Environ. Health Perspect. 120(10), 1425-1431 (2012).

15 Breitling LP, Yang R, Korn B, Burwinkel B, Brenner H. Tobacco-smoking-related differential DNA methylation: $27 \mathrm{k}$ discovery and replication. Am. J. Hum. Genet. 88(4), 450-457 (2011).

16 Elliott HR, Tillin T, Mcardle WL et al. Differences in smoking associated DNA methylation patterns in South Asians and Europeans. Clin. Epigenet. 6(1), 4 (2014).

17 Van Dijk SJ, Molloy PL, Varinli H, Morrison JL, Muhlhausler BS, Members of epiSCOPE. Epigenetics and human obesity. Int. J. Obes. 39(1), 85-97 (2015).

18 Godfrey KM, Sheppard A, Gluckman PD et al. Epigenetic gene promoter methylation at birth is associated with child's later adiposity. Diabetes 60(5), 1528-1534 (2011).

19 Ge K. Epigenetic regulation of adipogenesis by histone methylation. Biochim. Biophys. Acta 1819(7), 727-732 (2012).

20 Relton CL, Davey Smith G. Is epidemiology ready for epigenetics? Int. J. Epidemiol. 41(1), 5-9 (2012).

21 Wild CP, Scalbert A, Herceg Z. Measuring the exposome: a powerful basis for evaluating environmental exposures and cancer risk. Environ. Mol. Mutat. 54(7), 480-499 (2013). 